\title{
Envejecimiento demográfico urbano y sus repercusiones socioespaciales en México. Retos de la planeación gerontológica ${ }^{1}$
}

\author{
Diego Sánchez González²
}

\begin{abstract}
RESUMEN
El estudio analiza la evolución del proceso de envejecimiento demográfico urbano y sus repercusiones socioespaciales en México, a través de diferentes niveles de organización espacial, utilización y percepción espacial de las condiciones de habitabilidad para los adultos mayores: la escala federal, la estatal, la municipal y la metropolitana. La metodología parte de una amplia revisión bibliográfica y del análisis de datos estadísticos de fuentes oficiales. Los resultados advierten del avance del fenómeno del envejecimiento a distintas escalas, evidenciando una desigual distribución espacial, presentando un modelo polarizado basado en la concentración urbana y en la dispersión rural. Asimismo, se reflexiona sobre la preocupante inadecuación de las ciudades a las crecientes demandas de los adultos mayores, y se proponen estrategias de planeación gerontológica para abatir la desigualdad y potenciar el bienestar de la población de 60 años y más.
\end{abstract}

Palabras clave: Envejecimiento demográfico urbano, planeación gerontológica, calidad de vida, servicios sociales, áreas metropolitanas.

\begin{abstract}
The study analyzes the demographic evolution, pointing to the spatial repercussions of the aging process in Mexico, across different levels of spatial organization, utilization and spatial perception of the conditions of habitability for the major adults: the federal scale, the state one, the police officer and the metropolitan one. The methodology departs from a wide bibliographical review and from analyzing statistical information from official sources. The results notice from the advance of the phenomenon of the aging to different scales and with unequal spatial distribution, presenting a polarized model based on the urban concentration and on the rural dispersion. Likewise, the papers speculates about the inadequacy of the cities to meet the increasing demands of major adults, proposing a strategies of planning gerontology to bring down the inequality and to promote the well-being of the population of 60-year-old or more.
\end{abstract}

Key words: Demographic urban aging, planning gerontology, quality of life; social services, metropolitan areas.

Artículo recibido el 12 de marzo de 2007 y aceptado el 17 de septiembre de 2007.

2 Sistema Nacional de Investigadores de México (CONACYT), Sociedad Española de Geriatría y Ge- rontología e Instituto de Investigación en Ingeniería, Universidad Autónoma de Tamaulipas, Tampico, Tamaulipas (México).

E-mail: diegosanchezglez@hotmail.com 
En las últimas décadas México se ha vuelto un país urbano. La solución a los diversos problemas planteados por los distintos colectivos, como el de las personas adultas mayores, tiene su obligada plasmación espacial en nuestras ciudades. De ahí la necesidad de estudiarlas, percibirlas, recoger sus recuerdos y utilizarlos en pos de poder construir espacios más habitables para todos. Es necesario que los ciudadanos seamos más conscientes de la importancia de participar en la construcción y gestión de las ciudades.

La planeación gerontológica de los municipios urbanos y áreas metropolitanas obliga a la inclusión de nuevas aproximaciones teórico-metodológicas sobre el conocimiento de los distintos escenarios demográficos, socioeconómicos y políticos para la elaboración de políticas sociales eficaces que garanticen ciertos niveles de bienestar a la población adulta mayor (Kennedy \& Dejong, 1977; INSERSO, 1993; Sánchez, 2005a).

Diferentes investigaciones (Montes De Oca, 2003; Partida, 2005) señalan que el proceso de envejecimiento demográfico es una realidad a nivel mundial, regional y local. La creciente concentración de la población envejecida en las grandes áreas metropolitanas exige incluir dicha escala espacial en la planeación gerontológica. En la actualidad, las autoridades municipales son las encargadas de atender las necesidades de la heterogénea población de 60 años y más, sin embargo, la compleja realidad de las áreas metropolitanas exige promover líneas de investigación que fomenten el desarrollo social y asistencial de los adultos mayores a distintas escalas. Un juego de escalas que debe ser asumido e incorporado por las Administraciones y los profesionales para mejorar la atención de este sector de población.

El estudio presenta el avance y distribución espacial del envejecimiento demográfico en México, prestando especial atención al contexto urbano, y las alarmantes previsiones para las próximas décadas. Esta realidad poblacional sirve de base para abordar los principales problemas de vulnerabilidad que soportan hoy los adultos mayores en las ciudades (pobreza, desprotección de los servicios médicos y sociales, dependencia, soledad y vivienda). A través de la revisión bibliográfica se observa la falta reiterada de planeación gerontológica en México, así como la escasa presencia de expertos en los órganos de decisión de las cuestiones relativas a la vejez urbana.

El estudio concluye aportando algunas alternativas conceptuales y metodológicas para la mejora de la planeación gerontológica de las urbes y proponiendo un cambio radical e integral de crecimiento de las ciudades.

\section{Objetivo y metodología}

El objetivo general de la investigación es conocer la evolución reciente del proceso de envejecimiento demográfico urbano y su desigual distribución socioespacial en México. En este sentido, se analiza la distribución espacial de los adultos mayores por rango-tamaño de localidad a nivel municipal y de las entidades federativas, a nivel municipal y metropolitano. Con ello, se pretende comprender las causas y consecuencias que explican el modelo territorial del fenómeno demográfico a estudio.

A partir de los fuentes oficiales (INEGI, 2005), la investigación describe la problemática situación de las personas adultas mayores en las ciudades mexicanas, especialmente en las áreas metropolitanas, abordando aspectos como la vivienda, el acceso a los servicios médicos y sociales, y los equipamientos urbanos. El conocimiento resultante nos sirve para abrir el debate y reflexionar sobre las estrategias y políticas sociales que deben primar en la atención del adulto mayor en nuestras ciudades.

La metodología está basada en una amplia revisión bibliográfica, la consulta de bases de datos (INEGI, 2005; CONAPO, 2002) y la realización de cartografía temática a escalas estatal y municipal a partir de Sistemas de Información Geográfica. El trabajo responde no tanto al seguimiento de un método encaminado a realizar demostraciones, sino, más bien, a poner sobre la mesa de discusión un tema complejo y de gran relevancia: el proceso de envejecimiento urba- 
no y sus consecuencias geográficas en el plano social como económico, escasamente atendido por los investigadores y por la $\mathrm{Ad}$ ministración pública.

\section{Evolución, estructura y distribución espacial del envejecimiento demográfico}

La planeación gerontológica de los espacios urbanos exige tomar en cuenta el proceso de envejecimiento de la población de México para tener una visión preventiva (Sánchez, 2005b). El rápido avance del envejecimiento demográfico se atribuye principalmente a tres factores demográficos asociados: la caída de la tasa de fecundidad (Muñoz, 1987; Castillo y Vela, 2005); el descenso generalizado de la mortalidad, que ha significado el aumento de la esperanza de vida en todo el mundo, y los movimientos migratorios.

En este apartado se observan los cambios en la fecundidad, la mortalidad y en la estructura demográfica en México, así como la desigual distribución espacial del envejecimiento de la población en el territorio. Además, se apuntan los posibles escenarios del envejecimiento demográfico y sus repercusiones socioeconómicas y asistenciales (Menéndez, 2003; Gutiérrez y García, 2005).

El proceso de envejecimiento demográfico es un fenómeno universal sin precedentes, cuyas repercusiones sociales, políticas y económicas estamos empezando a conocer (Kinsella y Velkoff, 2002). Según algunos expertos (Instituto Nacional del Envejecimiento, 2000) el mayor crecimiento del envejecimiento de la población para el siglo XXI lo experimentarán los países en desarrollo, como México, lo que agravará la situación de pobreza y marginación que viven los adultos mayores en esos países. Todo apunta a que este fenómeno se convertirá en uno de los mayores problemas estructurales de la humanidad en el presente milenio (Jackson, 2005).

Entre los años 1970 y 2005, México ha sido uno de los países en desarrollo que más ha experimentado una acelerada transición demográfica, lo que ha permitido que hoy 8,3 millones de mexicanos tengan 60 años y más (INEGI, 2005). Los principales factores explicativos del avance del envejecimiento demográfico son: en primer lugar, la caída de la fecundidad (Mendoza, 1998), derivado de los efectos de la urbanización; la mejora en la salud, la educación y la incorporación de la mujer al trabajo, que en México ha pasado de 6,8 a 2,9 hijos por mujer (INEGI, 2005). En segundo lugar, el descenso de la mortalidad, sobre todo infantil (del 156,3\% al 30,9\%o), se explica por la expansión de la cobertura médica (Salgado, 1976), lo que ha propiciado un aumento de la esperanza de vida al nacer (en los varones de los 59 a los 72 años y en las mujeres de los 63 a los 77 años) (INEGI, 2005). Sin embargo, en México encontramos estados como Guerrero, Oaxaca y Chiapas donde persisten importantes desigualdades y la esperanza de vida es inferior a la media nacional (Montes de Oca, 2003). En tercer lugar, la migración nacional e internacional, sobre todo en México hacia Estados Unidos, de población joven activa, ha precipitado el avance del envejecimiento demográfico de las regiones rurales deprimidas, motivado por los efectos de la urbanización y la perdida de competitividad del sector primario.

Entre los años 1970 y 2000, en México una de las consecuencias más importantes de la transición demográfica ha sido el cambio en la estructura por edades de la población debido al aumento considerable del peso relativo de las personas adultas mayores (del 5,6 al 7\%) y la disminución del peso de los jóvenes (del 48 al 33\%), lo que está provocando un avance del envejecimiento demográfico absoluto y relativo (Figura № 1). En el año 2005 el aumento de población absoluta de 60 y más años alcanza los 8.338 .835 adultos mayores, es decir, el $7,1 \%$ de los casi 103,3 millones de habitantes; de los que alrededor de 4,4 millones son mujeres adultas mayores (53,3\%) y 3,9 varones adultos mayores (46,7\%). Asimismo, en el último quinquenio (2000-2005) cada año se han sumado 278.076 nuevos adultos mayores al colectivo de la tercera edad. Además, en el año 2005 la población de 75 años y más, aquella que presenta más riesgo de dependencia, está formada por más de 2,2 millones de personas, es decir, el $27 \%$ de los adultos mayores de este país (INEGI, 2005). 
Figura № 1

HISTORIA Y PROYECCIÓN DE LA POBLACIÓN RELATIVA SEGÚN GRUPO DE EDAD, MÉXICO $1950-2050$

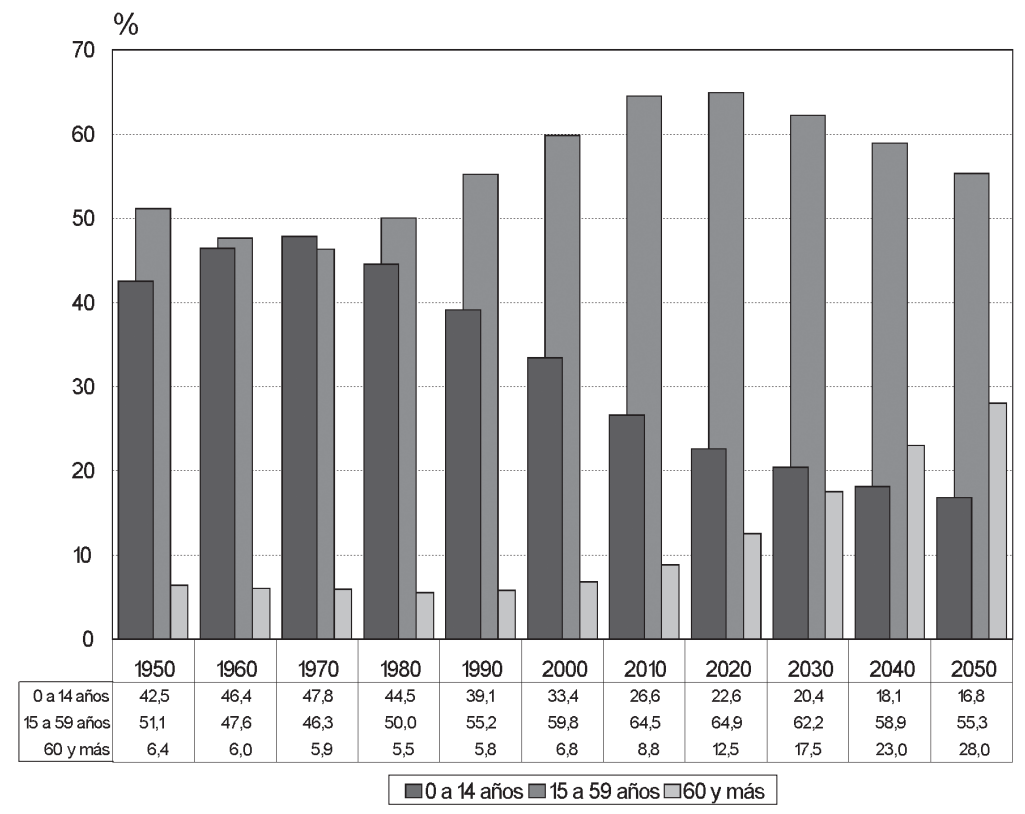

Fuente: Elaboración propia a partir de CONAPO (2002).

La Organización de las Naciones Unidas (ONU, 1985) prevé para el año 2050 que el proceso de envejecimiento demográfico tendrá especial relevancia en las regiones en desarrollo (Helpage International, 2002), como América Latina (Warnes y Horsey, 1988), donde la situación de pobreza y marginación de los adultos mayores es hoy especialmente complicada. Las previsiones para México indican que para el año 2030 la proporción de personas de 60 años y más será del $18 \%$ y en el año 2050 del $28 \%$ (CONAPO, 2002) (Figura № 1).

En México la desigual distribución espacial del fenómeno del envejecimiento es una realidad histórica y estructural motivada por el grado de avance de la transición demográfica regional y por la emigración (Negrete, 2001). Al mismo tiempo, se observan grandes coincidencias entre las zonas con baja densidad y elevados índices de envejecimiento, sobre todo en las regiones tradicionalmente agrícolas, que han padecido el efecto de llamada de la población activa joven hacia las grandes áreas urbanas, indus- triales y turísticas. Además, en las regiones rurales la permanencia de la población anciana se ha visto incrementada por la llegada de emigrantes de retorno de edades avanzadas a sus lugares de origen.

Entre los años 2000-2005 se observa el avance del envejecimiento demográfico absoluto y relativo en las áreas urbanas. La distribución de la población absoluta de 60 años y más por rango-tamaño de localidad arroja una marcada tendencia hacia la concentración urbana. Las ciudades han incrementado su población absoluta de la tercera edad, pasando de casi 4 a 4,9 millones de personas en sólo cinco años, lo que supone que en las localidades superiores a 15.000 habitantes vive el $58,4 \%$ del total (Cuadro № 1). Por su parte, en las localidades mixtas (entre 2.500 y 14.999 habitantes) residen 1,2 millones de adultos mayores, es decir, el 14,1\% del total. Asimismo, en las localidades rurales, aquellas menores de 2.500 habitantes, habitan algo menos de 2,3 millones de adultos mayores, el $27,4 \%$ (INEGI, 2005). 
Cuadro № 1

DISTRIBUCIÓN DE LA POBLACIÓN ABSOLUTA Y RELATIVA TOTAL Y ADULTA MAYOR SEGÚN RANGO-TAMAÑO DE LOCALIDAD, MÉXICO (AÑO 2005)

\begin{tabular}{|c|c|c|c|c|c|c|c|}
\hline \multirow[t]{2}{*}{$\begin{array}{l}\text { Rango-tamaño } \\
\text { de localidad }\end{array}$} & \multicolumn{2}{|c|}{ Población total } & \multicolumn{3}{|c|}{ Población de 60 años y más } & \multicolumn{2}{|c|}{$\begin{array}{l}\text { Población de } \\
70 \text { años y más }\end{array}$} \\
\hline & Total & $(\%)$ & Total & $(\%)$ & $\begin{array}{c}\text { Tasa de } \\
\text { envejecimiento }\end{array}$ & Total & $(\%)$ \\
\hline 1 a 2.499 habitantes & 24.276 .536 & 23,5 & 2.287 .442 & 27,4 & 9,5 & 1.071 .067 & 28,5 \\
\hline 2.500 a 14.999 Hab. & 14.130 .719 & 13,7 & 1.178 .424 & 14,1 & 8,5 & 552.993 & 14,7 \\
\hline 15.000 a $99.999 \mathrm{Hab}$. & 14.364 .335 & 13,9 & 1.074 .197 & 12,9 & 7,7 & 485.734 & 12,9 \\
\hline 100.000 y más Hab. & 50.491 .798 & 48,9 & 3.798 .772 & 45,6 & 7,8 & 1.648 .496 & 43,9 \\
\hline Total nacional & 103.263 .388 & 100,0 & 8.338 .835 & 100,0 & 8,3 & 3.758 .290 & 100,0 \\
\hline
\end{tabular}

Fuente: Elaboración propia a partir de INEGI (2005).

Hoy, México presenta un modelo polarizado de distribución espacial de la población que se agudiza en el caso de la población anciana: por un lado, la concentración urbana en las 26 áreas metropolitanas más grandes del país; aquellas con más de medio millón de habitantes concentran el 46,9\% de la población total y casi tres cuartas partes de la población de 60 y más años (73\%). A la inversa, la dispersión rural; en las más de 180 mil localidades rurales habita solo el $23,5 \%$ de la población total y el $27,4 \%$ de la población adulta mayor (INEGI, 2005).

En relación a la distribución de la población relativa de 60 años y más por rango-tamaño de localidad, se observa que entre los años 2000-2005 las localidades urbanas ven incrementado el peso relativo de los adultos mayores del 6,8 al $7,8 \%$. Por su parte, las localidades rurales han experimentado un envejecimiento demográfico más acusado del 8,1 al 9,5\% (INEGI, 2005).

La escala estatal nos muestra de nuevo una desigual distribución espacial del fenómeno del envejecimiento en el país, donde podemos diferenciar estados envejecidos demográficamente y afectados por la emigración, de aquellos otros menos envejecidos y de mayor atracción migratoria. Entre los años 2000-2005 los estados que experimentaron incrementos superiores al $1,4 \%$ en el envejecimiento de su población fueron Chiapas $(2,3 \%)$, Distrito Federal, Veracruz, Oaxaca y Sinaloa. A contrario, Coahuila,
Tlaxcala, Colima y Baja California Sur registraron incrementos inferiores al 0,5\%. En términos absolutos, en el año 2005 los estados con una mayor presencia de adultos mayores se localiza en el Estado de México, Distrito Federal, Veracruz y Jalisco, todos por encima del medio millón de habitantes, y que en su conjunto suponen el 35,8\% del total. A la inversa, los estados con menos adultos mayores se registra en Baja California Sur, Quintana Roo y Colima, todos inferiores a los 50 mil habitantes (INEGI, 2005).

Observando la Figura № 2, podemos identificar los estados con un mayor índice de envejecimiento demográfico en el año 2005, como son el Distrito Federal (10,2\%), Oaxaca, Zacatecas, Nayarit, Michoacán y Veracruz, que presentan tasas por encima del 9,4\%. Precisamente, estas entidades experimentan los efectos de la emigración y de los cambios en la transición demográfica (caída de la fecundidad y de la mortalidad), así como el retorno de los emigrantes de edades avanzadas. Por su parte, Quintana Roo (4,4\%), Baja California y Baja California Sur registran tasas por debajo del 6,5\%, favorecidas por el desarrollo del turismo de sol y playa y la llegada de población adulta joven de otras regiones del país. Además, el Estado de México $(6,6 \%)$ se ve rejuvenecido por la llegada de población joven procedente, sobre todo del Distrito Federal (INEGI, 2005), un estado afectado por un acusado proceso de especulación inmobiliaria que favorece la expulsión de las familias jóvenes (Negrete, 2003). 
Figura № 2

DISTRIBUCIÓN RELATIVA DE LA POBLACIÓN DE 60 Y MÁS AÑOS SEGÚN ESTADOS MÉXICO (AÑO 2005)

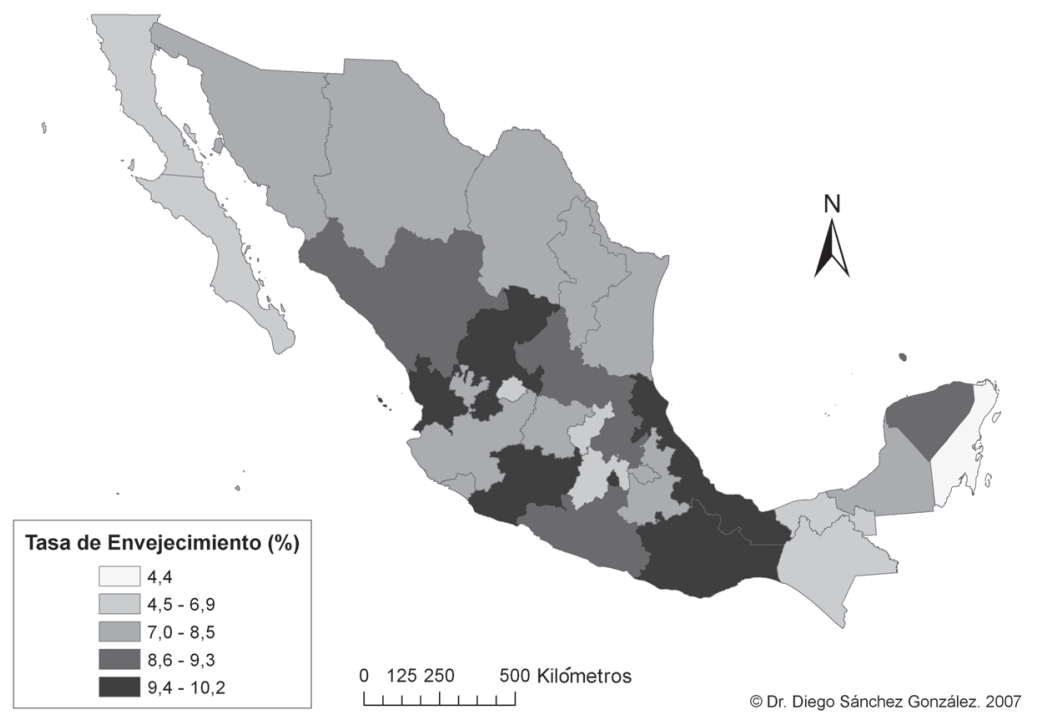

Fuente: Elaboración propia a partir de INEGI (2005).

Los cambios en la estructura de la población no se dan de manera homogénea en ambos sexos, la mayor esperanza de vida de las mujeres y la sobremortalidad de los varones deriva en una progresiva feminización de la población adulta mayor de México. La relación es más marcada conforme aumenta las edades de las cohortes: 88 varones por cada 100 mujeres adultas mayores de 60 años y más; y 83 varones por cada 100 mujeres adultas mayores de 75 años y más. A nivel estatal se agudiza la feminización de la tercera edad, con índices de masculinidad inferiores a 85 varones por cada 100 mujeres adultas mayores en los estados del centro del país, el Distrito Federal, Puebla, Estado de México y Aguascalientes. En cambio, los estados de Quintana Roo, Campeche y Chiapas, menos envejecidos demográficamente, presentan índices con una superioridad de varones sobre mujeres adultas mayores (INEGI, 2005).

Los cambios en la estructura por edad de la población en las entidades federativas se reflejan en el aumento de la relación de dependencia de adultos mayores, un indicador que permite comparar la relación entre la población jubilada (de 60 años y más) y la población activa (de 15 a 59 años), y que nos permite estimar la viabilidad de los sistemas de pensiones y otros gastos sociales que soporta actualmente la población activa. Entre los años 2000-2005 se ha producido un incremento del $1,4 \%$ a nivel nacional, que pasa de 12,4 a 13,8 adultos mayores por cada 100 personas en edad de trabajar (INEGI, 2005). A nivel estatal, dicho indicador presenta marcadas diferencias entre los estados durante ese corto periodo: por una parte, aquellos que registran incrementos por encima del 2\% (Distrito Federal, Veracruz, Sinaloa y Guerrero); y de otra, aquellos con crecimientos inferiores al 0,6\% (Baja California Sur, Tlaxcala, Baja California y Yucatán). Asimismo, en el año 2005 la relación de dependencia demográfica de adultos mayores es más alta en Oaxaca $(17,7 \%)$, Zacatecas $(17,3 \%)$, Michoacán $(16,7 \%)$ y Nayarit $(16,5 \%)$; en tanto que, resulta menor en Quintana Roo (7\%), Baja California (9,9\%), Baja California Sur (10\%) y Estado de México, con 10,7 adultos mayores por cada 100 personas en edad de trabajar, debido al rejuvenecimiento por la llegada de población inmigrante joven activa (INEGI, 2005). 
En el juego de escalas para la comprensión espacial del proceso de envejecimiento demográfico, cobra especial significado el nivel municipal, que refleja un modelo polarizado de concentración urbana y dispersión rural. Los efectos desiguales de la migración en los municipios mexicanos tienen su plasmación en la distribución absoluta y relativa del fenómeno del envejecimiento. En números absolutos, en el año 2005 la población de 60 años y más rebasa los 99,5 mil habitantes en los municipios del Estado de Jalisco (Guadalajara, 157,7 mil hab.), Distrito Federal (Iztapalapa y Gustavo A. Madero), Nuevo León (Monterrey), Puebla (Puebla) y Estado de México (Nezahualcóyotl y Ecatepec). Estos siete municipios forman parte de las ciudades más pobladas del país y concentran a más del 10,2\% del total de adultos mayores. A la inversa, en los municipios rurales de los estados de Oaxaca (Santa Magdalena ${ }^{3}$, Santiago Tepetlapa y Santo Domingo), Sonora (San Javier, Onavas y San Felipe) y Puebla (La Magdalena y San Juan Atzompa) residen menos de 100 adultos mayores (INEGI, 2005). Estos municipios rurales padecen una fuerte emigración de la población joven y un acusado envejecimiento.

En términos relativos, se registra un menor índice de envejecimiento en los municipios turísticos del Estado de Quintana Roo, como Solidaridad (2,3\%) y Benito Juárez (3,3\%); seguidos por los municipios del área metropolitana de Monterrey (Nuevo León), como Juárez (3,1\%), Apodaca $(3,2 \%)$ y General Escobedo (3,7\%) (Figura № 3). Aquí se observa con claridad cómo el efecto llamada de las áreas turísticas y urbana-industriales redundan en menores tasas de envejecimiento, beneficiadas por la llegada de población inmigrante activa joven. Por el contrario, los municipios rurales del Estado de Oaxaca, como Santiago Tepetlapa $(39,1 \%)$ y Santiago Nejapilla $(36,9 \%)$ tienen la mayor proporción de población adulta

\footnotetext{
3 En el año 2005 el municipio rural de Santa Magdalena (Oaxaca) es al mismo tiempo la entidad municipal de menor atracción demográfica del país (102 habitantes) y tiene la menor cantidad de adultos mayores (23 habitantes).
}

mayor, seguidos de Axutla $(30,1 \%)$ y San Miguel Ixitlán $(29,1 \%)$ (Estado de Puebla), Dr. Belisario Domínguez (28,6\%) (estado de Chihuahua), y El Plateado de Joaquín Amaro $(27,2 \%)$ (Estado de Zacatecas) (INEGI, 2005).

La migración es uno de los principales factores explicativos de la desigual distribución de la población relativa de 60 años y más según los municipios del país. Precisamente, los municipios de expulsión, sobre todo rurales, presentan tasas de envejecimiento, por encima del $8,5 \%$, mientras que los municipios de atracción tienen un peso relativo por debajo del 5,5\%. El mayor peso relativo de los adultos mayores en los municipios rurales de expulsión explica mayores tasas de dependencia demográfica de la tercera edad por municipios, destacando Santiago Tepetlapa, Santiago Nejapilla, San Miguel Tulancingo y San Baltasar (estado de Oaxaca), con más de 80 adultos mayores por cada 100 personas en edad de trabajar. En tanto que Solidaridad y Benito Juárez (Quintana Roo) y Apodaca y Juárez (Nuevo León) presentan una relación de dependencia inferior a los 5 adultos mayores por cada 100 personas en edad de trabajar (INEGI, 2005).

En general, se está produciendo un avance del envejecimiento demográfico urbano en términos absolutos y relativos, lo que debe marcar las políticas sociales y la planeación gerontológica de este país. En este sentido, resulta especialmente interesante observar el proceso de concentración de la población adulta mayor a nivel metropolitano, en especial, las principales áreas metropolitanas de México, aquellas que superan el medio millón de habitantes (INEGI, 2005).

Entre los años 2000-2005 las principales áreas metropolitanas experimentan un incremento global de 6,8 millones de habitantes, es decir, un 16,3\%. Aquí, destacamos a Villahermosa (Tabasco), Toluca (estado de México) y Querétaro (Querétaro), todas con incrementos superiores al $60 \%$ y, principalmente, motivados por la expansión de dichas áreas metropolitanas y el aumento del número de municipios que las forman, así como la consiguiente suma de sus efectivos. 
Figura № 3

DISTRIBUCIÓN RELATIVA DE LA POBLACIÓN DE 60 Y MÁS AÑOS SEGÚN MUNICIPIOS MÉXICO (AÑO 2005)

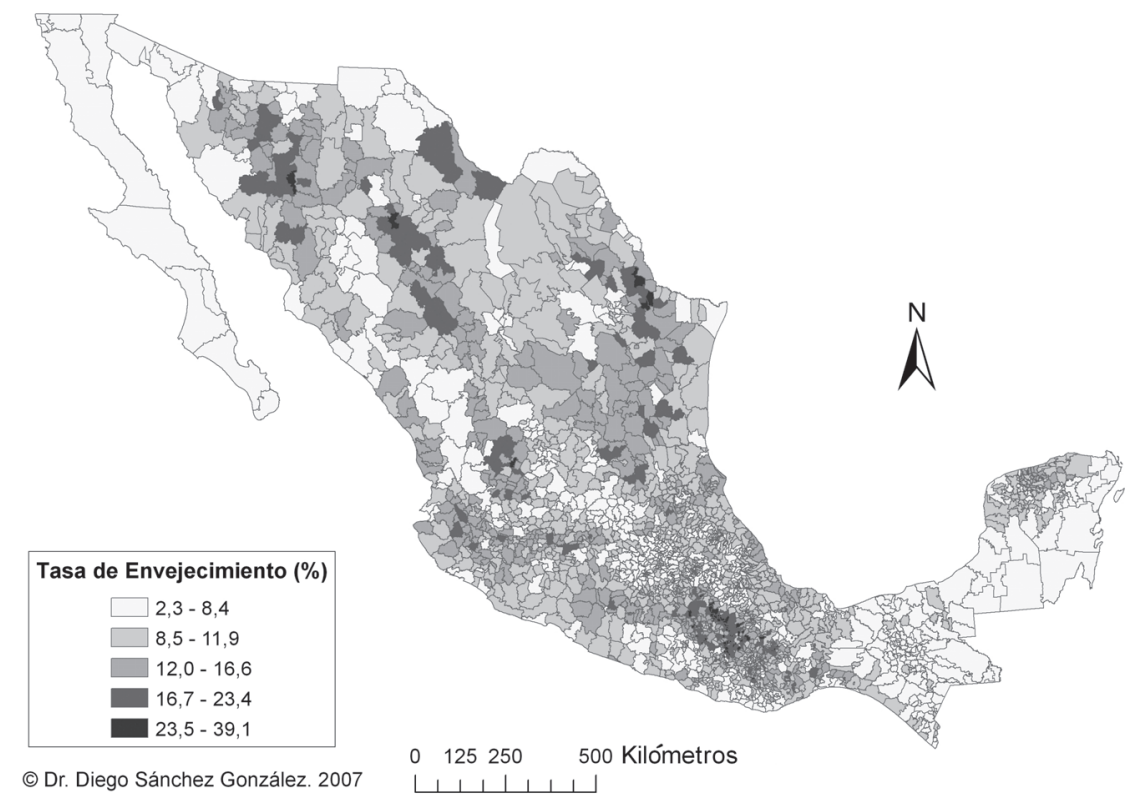

Fuente: Elaboración propia a partir de INEGI (2005).

Por el contrario, Acapulco fue la única gran área metropolitana que tuvo un ligero incremento negativo $(-0,6 \%)$ (INEGI, 2005), motivado por el estancamiento del sector turístico en los últimos años, unido al desarrollo de otros destinos turísticos (Cancún, Mazatlán, Los Cabos).

En datos absolutos, en el año 2005 se observa que casi uno de cada dos mexicanos vivía en una área metropolitana $(48,4$ millones de personas) (INEGI, 2005) (Cuadro № 2). Aquí, destacan por su volumen la Ciudad de México (19,2 millones de Hab.), Guadalajara (4,1 millones de Hab.) y Monterrey (3,7 millones de Hab.), tradicionalmente los tres grandes centros urbanos e industriales del país. En menor medida, encontramos que el área metropolitana de Oaxaca (504.159 Hab.) se ha visto afectada por la emigración rural y urbana hacia otras regiones más dinámicas.

En general, se observa un avance del envejecimiento en las áreas metropolitanas asociado a la evolución de la transición demográfica regional y urbana, y los procesos migratorios. Además, la migración es una consecuencia de los procesos especulativos de suelo urbano, que expulsa a las familias jóvenes a la periferia de las ciudades; así como del retorno de grupos de población de edad avanzada.

Entre los años 2000-2005 en las áreas metropolitanas se produce un incremento general de 828.431 adultos mayores, es decir, un $30,4 \%$. Esto supone que la población de 60 años y más crece al doble del ritmo de la población general en las grandes urbes. Aquí, destacan Acapulco (Guerrero), Xalapa (Veracruz), Cuernavaca (Morelos) y Ciudad de México (Distrito Federal), todas por encima del $1,1 \%$ de incremento, y afectadas por el fenómeno de la expulsión de población joven (INEGI, 2005) (Cuadro № 2). En el otro extremo, encontramos las áreas metropolitanas de Reynosa-Río Bravo (Tamaulipas), Tijuana (Baja California) y Juárez (Chihuahua), todas por debajo del 0,6\%, y ubicadas en la 
En términos absolutos (Cuadro № 2), en el año 2005 en las áreas metropolitanas viven más de 3,5 millones de personas adultas mayores, lo que representa el $42,7 \%$ del total. El dato justifica la necesidad de implementar dicha escala territorial en la planeación de los servicios sociales y urbanos

Cuadro № 2

DISTRIBUCIÓN TERRITORIAL DE LA POBLACIÓN TOTAL Y DE 60 AÑOS Y MÁS SEGÚN ÁREA METROPOLITANA, MÉXICO (AÑOS 2000-2005)

\begin{tabular}{|c|c|c|c|c|c|c|c|c|}
\hline \multirow[b]{2}{*}{$\begin{array}{c}\text { Área } \\
\text { Metropolitana }\end{array}$} & \multicolumn{3}{|c|}{2000} & \multicolumn{3}{|c|}{2005} & \multicolumn{2}{|c|}{ 2000-2005 } \\
\hline & $\begin{array}{c}\text { Población } \\
\text { total } \\
\text { (absoluto) }\end{array}$ & $\begin{array}{l}\text { Grupo } \\
60 \text { años } \\
\text { y más } \\
\text { (absoluto) }\end{array}$ & $\begin{array}{c}\text { Grupo } 60 \\
\text { años y } \\
\text { más }(\%)\end{array}$ & $\begin{array}{c}\text { Población } \\
\text { total } \\
\text { (absoluto) }\end{array}$ & $\begin{array}{c}\text { Grupo } 60 \\
\text { años y } \\
\text { más } \\
\text { (absoluto) }\end{array}$ & $\begin{array}{c}\text { Grupo } \\
60 \text { años } \\
\text { y más (\%) }\end{array}$ & $\begin{array}{l}\text { Población } \\
\text { total } \\
\text { Incremento } \\
\text { anual }(\%)\end{array}$ & $\begin{array}{c}\text { Grupo } 60 \\
\text { años y más } \\
\text { Incre- } \\
\text { mento }(\%)\end{array}$ \\
\hline Acapulco & 791.558 & 51.489 & 6,7 & 786.830 & 61.709 & 8,17 & $-0,12$ & 1,49 \\
\hline Aguascalientes & 707.516 & 42.345 & 6,0 & 805.666 & 53.282 & 6,73 & 2,77 & 0,70 \\
\hline Cancún & 431.128 & 11.160 & 2,6 & 586.288 & 16.611 & 3,38 & 7,20 & 0,75 \\
\hline Chihuahua & 696.495 & 52.827 & 7,7 & 784.882 & 63.402 & 8,60 & 2,54 & 0,94 \\
\hline Ciudad de México & 17.308 .562 & 1.182 .727 & 7,0 & 19.231 .829 & 1.514 .760 & 8,21 & 2,22 & 1,16 \\
\hline Cuernavaca & 672.719 & 49.404 & 7,6 & 787.556 & 66.361 & 8,91 & 3,41 & 1,28 \\
\hline Guadalajara & 3.477 .101 & 229.465 & 6,7 & 4.095 .853 & 294.956 & 7,50 & 3,56 & 0,83 \\
\hline Juárez & 1.228 .849 & 61.942 & 5,4 & 1.313 .338 & 72.670 & 5,93 & 1,38 & 0,54 \\
\hline La Laguna & 771.939 & 54.944 & 7,2 & 1.110 .890 & 86.489 & 7,91 & 8,78 & 0,73 \\
\hline León & 1.369 .418 & 78.877 & 5,8 & 1.425 .210 & 89.428 & 6,41 & 0,81 & 0,60 \\
\hline Mérida & 743.983 & 62.123 & 8,4 & 897.740 & 79.016 & 9,09 & 4,13 & 0,67 \\
\hline Monterrey & 3.161 .509 & 211.510 & 6,8 & 3.664 .331 & 272.796 & 7,61 & 3,18 & 0,85 \\
\hline Morelia & 659.940 & 46.243 & 7,1 & 735.624 & 56.722 & 8,14 & 2,29 & 1,07 \\
\hline Oaxaca & 437.634 & 29.292 & 6,7 & 504.159 & 37.127 & 7,67 & 3,04 & 0,93 \\
\hline Puebla-Tlaxcala & 2.104 .935 & 140.326 & 6,8 & 2.109 .049 & 151.821 & 7,48 & 0,04 & 0,63 \\
\hline Querétaro & 554.707 & 29.325 & 5,4 & 918.100 & 54.876 & 6,23 & 13,10 & 0,87 \\
\hline Reynosa-Río Bravo & 524.692 & 32.025 & 6,2 & 633.730 & 38.326 & 6,45 & 4,16 & 0,27 \\
\hline Saltillo & 593.909 & 34.335 & 5,8 & 725.259 & 48.949 & 6,89 & 4,42 & 1,04 \\
\hline San Luis Potosí & 798.782 & 51.014 & 6,5 & 957.753 & 67.491 & 7,17 & 3,98 & 0,71 \\
\hline Tampico-Panuco & 590.119 & 47.116 & 8,0 & 803.196 & 71.740 & 9,08 & 7,22 & 1,04 \\
\hline Tijuana & 1.274 .240 & 55.804 & 4,8 & 1.483 .992 & 72.396 & 5,37 & 3,29 & 0,53 \\
\hline Toluca & 950.198 & 50.074 & 5,5 & 1.610 .786 & 96.578 & 6,24 & 13,90 & 0,70 \\
\hline Tuxtla Gutiérrez & 510.653 & 27.861 & 5,5 & 576.872 & 36.298 & 6,45 & 2,59 & 0,91 \\
\hline Veracruz & 554.496 & 47.396 & 8,6 & 702.394 & 65.857 & 9,62 & 5,33 & 0,97 \\
\hline Villahermosa & 357.669 & 20.587 & 5,8 & 644.629 & 41.134 & 6,53 & 16,05 & 0,70 \\
\hline Xalapa & 392.447 & 28.829 & 7,4 & 545.567 & 46.676 & 8,73 & 7,80 & 1,30 \\
\hline
\end{tabular}

Fuente: Elaboración propia a partir de INEGI (2000-2005). 
(INEGI, 2005). Por el volumen de la población de 60 años y más, destaca la Ciudad de México (Distrito Federal) (1,5 millones de Hab.), Guadalajara (Jalisco) (295 mil Hab.) y Monterrey (Nuevo León) (272 mil Hab.). Las tres áreas metropolitanas en su conjunto suman más de 2 millones de personas de 60 años y más, es decir, uno de cada cuatro adultos mayores de este país (Montes de Oca, 1998). En cambio, en Cancún (Quintana Roo) residen 16.611 adultos mayores, lo que refleja el dinamismo del sector turístico y su influencia en la estructura de la población (INEGI, 2005).

En datos relativos (Cuadro № 2), el área metropolitana de Veracruz (Veracruz), Mérida (Yucatán) y Tampico-Panuco (Tamaulipas-Veracruz) presentan la mayor proporción de población adulta mayor, todas por encima del $9 \%$, y afectadas por el avance de la transición demográfica. A la inversa, una vez más, Cancún se convierte en la que tiene el menor porcentaje $(3,4 \%)$, debido a su mayor atracción de población adulta joven por el desarrollo turístico (INEGI, 2005).

En la razón de dependencia en la vejez destacan León (Guanajuato) (68), Aguascalientes (Aguascalientes) (66) y Acapulco (Guerrero), con 65 adultos mayores por cada 100 personas en edad de trabajar. Al revés, Veracruz (Veracruz) (53), Cancún (Quintana Roo) (53) y Villahermosa (Tabasco) con 54 adultos mayores por cada 100 personas en edad de trabajar (INEGI, 2005). De estos datos se desprende que las tasas más altas de dependencia en la vejez se asocian a áreas metropolitanas con procesos de envejecimiento demográfico más acusados, mientras que las áreas que registran menores tasas se ven beneficiados por una migración de atracción.

En síntesis, el contexto demográfico, presentado a distintas escalas geográficas, refleja una desigual distribución espacial de la población adulta mayor en México. El modelo polarizado de la población basado en la concentración urbana, favorecido por las localidades de atracción, y la dispersión rural por las localidades de expulsión, explican el grado de avance del envejecimiento de la población.
Hoy vemos con preocupación como tres de cada cuatro adultos mayores se localizan en las localidades urbanas, de preferencia en las grandes áreas metropolitanas. Estas metrópolis, escasamente planeadas, se enfrentan al reto de ofrecer calidad de vida a sus residentes, una cuestión que se vuelve aún más compleja en el caso de los adultos mayores y las personas dependientes. Ya que dichos espacios urbanos no reúnen las condiciones mínimas de habitabilidad ni los servicios básicos para estos ciudadanos longevos.

En las zonas rurales, con un envejecimiento relativo mayor, fruto de las oleadas emigratorias, también deberán ser muy tenidas en cuenta por las políticas sociales de los distintos estados. Aquí, defendemos el desarrollo ordenado de las ciudades, como motor de crecimiento en sinergia con su región y, por el otro, enfrentar las profundas desigualdades de la población adulta mayor urbana y rural.

Surgen serias dudas sobre las posibilidades reales de atender adecuadamente las demandas crecientes de tan numerosa población envejecida, en plena progresión, que vivirá en las ciudades. Se hace indispensable un conocimiento profundo y previo de la situación de las personas adultas mayores de dichos espacios con la finalidad de posibilitar una adecuada planeación de los servicios necesarios. El gran reto de nuestra sociedad, cada vez más urbana y envejecida, está en superar la pobreza y la exclusión social que seguirán padeciendo los adultos mayores en el presente milenio. Es prioritario favorecer una mejora de la calidad de vida de la población envejecida que vive en nuestras ciudades.

\section{Repercusiones socioespaciales del envejecimiento demográfico urbano en México}

Las ciudades registran graves problemas de habitabilidad para la población (Harvey, 1973; Castells, 1978; Gilbert y Gugler, 1992). Diferentes estudios (Compán y Sánchez, 2005) revelan que la ciudad es un es- 
pacio que no reúne las condiciones de habitabilidad para las personas adultas mayores. Un lugar inhóspito que excluye al anciano, lo que favorece su dependencia y aislamiento social.

A continuación, pasamos a conocer la problemática situación de las personas adultas mayores en las ciudades mexicanas, a nivel municipal y, sobre todo, de las áreas metropolitanas. Aquí, describimos la realidad del grupo de adultos mayores en situación de vulnerabilidad y en su contexto urbano y metropolitano a través de aspectos básicos como: la educación, la cobertura de los servicios sociales y de salud, la pobreza, la soledad y la vivienda.

México presenta cierto rezago educativo, que es especialmente significativo en el caso de la población de 60 años y más, donde el $28,4 \%$ es analfabeta (no sabe leer ni escribir) y más del $63 \%$ es analfabeta funcional (sin estudios terminados), tasas que se incrementan con la edad y, sobre todo, entre las mujeres de 75 años y más (INEGI, 2005). En la actualidad, las localidades urbanas concentran al $16,5 \%$ de los adultos mayores analfabetos y a uno de cada dos que no tiene estudios terminados $(47,8 \%)$, mientras que las áreas metropolitanas registran el $15,8 \%$ y el $21,4 \%$, respectivamente. Aquí, por su alta población analfabeta, destacan Acapulco (Guerrero) (38\%) y Tuxtla Gutiérrez (Chiapas) (30,5\%); en tanto que Chihuahua y Juárez (Chihuahua), La Laguna (Coahuila) y Monterrey (Nuevo León), localizadas al norte del país, presentan tasas por debajo del $11,5 \%$ (INEGI, 2005).

La educación define la vida laboral y condiciona el acceso posterior al tipo de pensión y al seguro médico. En las localidades de 100.000 habitantes y más, solo el $36,5 \%$ de las personas de 60 y más años siguen trabajando después de la edad de jubilación, de las cuales, solo una de cada dos tiene un empleo formal y está asegurada (INEGI, 2005). El dato refleja que la mitad de los patrones de las empresas incumplen las leyes de trabajo, no proporcionándoles prestaciones laborales a sus trabajadores más longevos. Asimismo, se observan diferencias significativas según sexo, los hom- bres asegurados por derecho a pensión son el $31,4 \%$ y por beneficiarios del titular el $26,4 \%$, mientras las mujeres son de $15,1 \%$ y $58,8 \%$, respectivamente.

La cobertura de servicios de salud es factor importante en el bienestar del adulto mayor, ya que presentan tasas superiores de morbilidad y dependencia, las cuales se van agudizando conforme avanza la edad, sobre todo entre las mujeres de 75 años y más. En el año 2005 la población adulta mayor no derechohabiente (aquella que no tiene garantizado el acceso a los servicios médicos y/u otras prestaciones) es del 44,1\%. Una situación que se vuelve dramática para casi 3,7 millones de adultos mayores que no cuentan con servicios de salud. En las localidades urbanas, la desprotección afecta al 30,4\% de los que han cumplido 60 años y más, y al 30,7\% de los que residen en áreas metropolitanas, especialmente en Toluca (Estado de México) (50,4\%), Tuxtla Gutiérrez (Chiapas) $(46,4 \%)$, Acapulco (Guerrero) $(44,7 \%)$ y Oaxaca (Oaxaca) $(44,5 \%)$; y por el contrario, se registran tasas por debajo del 20\% en Chihuahua (Chihuahua), Monterrey (Nuevo León) y Mérida (Yucatán) (INEGI, 2005) (Cuadro № 3). Aquí, se observa una correlación significativa del $69 \%(r=0,69097)$ que nos indica que, a medida que aumenta el porcentaje de adultos mayores analfabetos, se incrementa el porcentaje de personas sin cobertura médica en las áreas metropolitanas del país.

En México, la familia continúa siendo la principal institución responsable del cuidado de los adultos mayores. Sin embargo, los cambios en la estructura familiar, la incorporación de la mujer al trabajo y la especulación inmobiliaria, expulsando a las familias jóvenes a viviendas sociales de reducidas dimensiones y localizadas en la periferia de las ciudades, imposibilitan el mantenimiento de la ayuda informal y fomentan la soledad en la vejez. Según estudios de INEGI (2005), el 16,8\% de los adultos mayores que viven solos tienen discapacidad, de los cuales, más del $60 \%$ no cuenta con ningún tipo de prestación y ayuda económica, médica, social y asistencial. 
Cuadro № 3

DISTRIBUCIÓN TERRITORIAL DE LA POBLACIÓN DE 60 AÑOS Y MÁS SEGÚN INDICADORES DE VULNERABILIDAD SOCIAL Y ÁREA METROPOLITANA, MÉXICO (AÑO 2005)

\begin{tabular}{|c|c|c|c|c|}
\hline Área Metropolitana & $\begin{array}{l}\text { Grupo } 60 \\
\text { años y más } \\
\text { (absoluto) }\end{array}$ & $\begin{array}{c}\text { No sabe leer } \\
\text { ni escribir } \\
(\%)\end{array}$ & $\begin{array}{c}\text { No derecho- } \\
\text { habiente } \\
(\%)\end{array}$ & $\begin{array}{c}\text { Adultos mayores } \\
\text { viviendo solos } \\
(\%)\end{array}$ \\
\hline Acapulco & 61.709 & 38,0 & 44,7 & 11,4 \\
\hline Aguascalientes & 53.282 & 15,2 & 21,2 & 9,2 \\
\hline Cancún & 16.611 & 18,4 & 34,7 & 8,3 \\
\hline Chihuahua & 63.402 & 6,5 & 16,9 & 11,9 \\
\hline Ciudad de México & 1.514 .760 & 13,6 & 30,7 & 8,4 \\
\hline Cuernavaca & 66.361 & 22,5 & 39,6 & 11,3 \\
\hline Guadalajara & 294.956 & 14,4 & 32,0 & 8,9 \\
\hline Juárez & 72.670 & 10,4 & 31,7 & 11,6 \\
\hline La Laguna & 86.489 & 10,8 & 22,4 & 9,4 \\
\hline León & 89.428 & 28,7 & 30,3 & 6,7 \\
\hline Mérida & 79.016 & 13,4 & 19,9 & 9,4 \\
\hline Monterrey & 272.796 & 11,4 & 16,9 & 7,7 \\
\hline Morelia & 56.722 & 22,2 & 42,3 & 9,1 \\
\hline Oaxaca & 37.127 & 23,2 & 44,5 & 7,3 \\
\hline Puebla-Tlaxcala & 151.821 & 18,9 & 39,1 & 8,0 \\
\hline Querétaro & 54.876 & 24,7 & 30,9 & 7,9 \\
\hline Reynosa-Río Bravo & 38.326 & 18,0 & 34,3 & 10,9 \\
\hline Saltillo & 48.949 & 15,0 & 21,4 & 8,7 \\
\hline San Luis Potosí & 67.491 & 16,5 & 25,3 & 9,2 \\
\hline Tampico-Panuco & 71.740 & 14,7 & 26,1 & 10,7 \\
\hline Tijuana & 72.396 & 12,5 & 42,2 & 10,9 \\
\hline Toluca & 96.578 & 29,0 & 50,4 & 7,0 \\
\hline Tuxtla Gutiérrez & 36.298 & 30,5 & 46,4 & 8,0 \\
\hline Veracruz & 65.857 & 15,5 & 29,0 & 11,1 \\
\hline Villahermosa & 41.134 & 23,5 & 27,8 & 8,8 \\
\hline Xalapa & 46.676 & 21,1 & 38,3 & 10,5 \\
\hline Total & 3.557 .471 & 15,8 & 30,7 & 8,8 \\
\hline
\end{tabular}

Fuente: Elaboración propia a partir de INEGI (2000-2005).

Hoy, la soledad afecta a más 817 mil adultos mayores en este país, es decir, el $9,8 \%$ del total. En términos relativos, la soledad en la vejez la padece el $9,3 \%$ de los que residen en las localidades urbanas y el $8,8 \%$ de los que viven en las áreas metropolitanas. Este indicador tiene tasas superiores al $11 \%$ en Chihuahua y Juárez (Estados de Chihuahua) y Cuernavaca (Morelos); mientras que se localizan porcentajes inferiores al 7\% en León (Guanajuato) y Toluca (Estado de México) (Cuadro № 3).

La problemática de muchas viviendas habitadas por ancianos se debe a la autoconstrucción, que es una estrategia que emplean los sectores más desfavorecidos de la sociedad. Precisamente, la exclusión de los programas de vivienda de interés social (Romero; Hernández, y Acevedo, 2005), como 
las del Instituto del Fondo Nacional para la Vivienda de los Trabajadores (INFONAVIT), viene determinada por la exigencia de una serie de requisitos (empleo formal, ahorro e ingresos) que no pueden ser cubiertos por la población adulta mayor con escasos recursos. La exclusión de los programas de viviendas sociales refuerza la marginación de millones de ancianos pobres y sus familias. Además, los programas resultan ser insuficientes y se alejan de los parámetros de confort, funcionalidad y materiales de sus destinatarios. Por el contrario, el hacinamiento y segregación son característicos de las unidades habitacionales de los ancianos, lo que conlleva aislamiento y exclusión social (Schteingart y Graizbord, 2000).

Las viviendas de los adultos mayores presentan deficiencias notables en cuanto a los materiales de construcción. Observamos que el 32,2\% están construidas con materiales no duraderos (hojalata, chapa, cartón, madera); y casi el $10 \%$ no tienen excusado (INEGI, 2005). Además, el $20 \%$ de las viviendas habitadas por ancianos tienen techo de lámina de asbesto, un material que es dañino para la salud. Hoy, 992.080 viviendas ocupadas por adultos mayores tienen piso de tierra, es decir, el 11,9\% del total, lo que dificulta el aseo del lugar y facilita la propagación de enfermedades a través de parásitos (cucarachas, ratas). En términos relativos, esta problemática afecta al 3,2\% de la población de 60 años y más en las localidades urbanas, porcentaje que se va incrementando entre las cohortes más avanzadas y las mujeres de 80 años y más (INEGI, 2005).

En México casi un millón de adultos mayores no dispone de agua entubada en el domicilio, esto es, el $11,5 \%$ del total, localizados, principalmente, en las localidades rurales. En términos relativos, el 2,8\% de la población de 60 y más años en las localidades urbanas y el 3,5\% en las áreas metropolitanas se ve afectado por la falta de agua, sobresaliendo Acapulco (Guerrero) (21\%) y Tuxtla Gutiérrez (Chiapas) (13,9\%), y en menor medida en La Laguna (Coahuila) $(0,8 \%)$ y Valle de México (Distrito Federal) (1,5\%).

Los domicilios sin electricidad habitados por adultos mayores se contabilizan en más de $228 \mathrm{mil}$, lo que representa el $2,8 \%$ del total, localizados en áreas rurales. En datos relativos, la problemática solo afecta al $0,5 \%$ de los adultos mayores de localidades urbanas y de áreas metropolitanas, destacando Reynosa (Tamaulipas) y Tampico-Panuco (Tamaulipas-Veracruz), ambas por encima $2,2 \%$; a la inversa, se registran porcentajes por debajo del $3 \%$ en Valle de México (Distrito Federal), Guadalajara (Jalisco) y Monterrey (Nuevo León).

Las viviendas sin drenaje suman más de 1,1 millones, en su mayoría ubicadas en localidades rurales, afectando al $13,5 \%$ de la población de 60 años y más del país. En términos relativos, el problema afecta al 1,6\% de los adultos mayores en las localidades urbanas y al 2,4\% en las áreas metropolitanas, destacando, con tasas superiores al 11,2\%, Reynosa (Tamaulipas) y Tampico-Panuco (Tamaulipas-Veracruz), y Acapulco (Guerrero). Encontramos tasas inferiores al 1\% en Guadalajara (Jalisco), Monterrey (Nuevo León), Chihuahua (Chihuahua) y Ciudad de México (Distrito Federal).

Los indicadores de vulnerabilidad social presentados para la población de 60 años y más permiten observar que los factores socioeconómicos (educación, profesión) durante la vida laboral condicionan la situación del adulto mayor en la vejez (seguro médico, vivienda). A nivel de áreas metropolitanas se distingue que aquellas más dinámicas, tanto demográficas como socioeconómicas (industria, turismo) (regiones de atracción), localizadas, sobre todo, en la frontera norte (Monterrey) y en Cancún, van a favorecer menores porcentajes de adultos mayores en riesgo de padecer vulnerabilidad. Las áreas menos dinámicas y envejecidas (regiones de expulsión), localizadas principalmente al sur del país (Oaxaca, Tuxtla Gutiérrez), repercute en un mayor peso relativo de la vulnerabilidad de la población anciana.

En México el papel de los municipios urbanos es esencial en la atención directa de las personas adultas mayores. En este sentido, la creciente concentración de la población envejecida en las ciudades, especialmente, en las grandes áreas metropolitanas, obliga a incluir dichas escalas en los nuevos diseños de la planeación urbana y gerontológica. 
Diferentes investigadores (Oates, 1972) defienden que los gobiernos municipales deben estar principalmente a cargo de la provisión de bienes públicos locales, pues son estos los que deben decidir qué bienes y servicios han de producir y cómo han de emplear sus recursos, asimismo, tienen mejor información sobre las distintas preferencias locales y, sobre todo, conocen más directamente las necesidades y demandas de la población. En este sentido, el alto endeudamiento y la escasa capacidad económica de los gobiernos municipales explican, en buena medida, la política y gestión local seguida en muchos municipios y destinada a priorizar algunas de las grandes necesidades en infraestructuras básicas, obligando a descuidar otros apartados sociales y asistenciales fundamentales para los adultos mayores. Ciertamente, los estudios (Boltvinik y Damián, 2001) indican que en México en los municipios de 100.000 habitantes y más se registran mayores tasas de marginalidad y pobreza extrema entre la población adulta mayor, demasiadas veces ignorada por las autoridades municipales y, en ocasiones, por los propios familiares que incurren en desamparo.

El debate se abre sobre la capacidad de los municipios mexicanos para ofrecer respuestas válidas a los ciudadanos más longevos. Un escenario plagado de dudas, ya que muchos ayuntamientos presentan un alto grado de endeudamiento, lo que repercute negativamente en la calidad de los servicios médicos, sociales y asistenciales ofrecidos a las personas adultas mayores. El reto de los gobiernos municipales será comprender la verdadera dimensión del envejecimiento demográfico y sus repercusiones socioespaciales a nivel regional, metropolitano, municipal e intramunicipal (feminización, vulnerabilidad, dependencia, inequidad social). Entre los desafíos que enfrentan los municipios de las áreas metropolitanas del país está el integrar las políticas públicas destinadas al adulto mayor y buscar soluciones consensuadas basadas en el reparto equitativo del gasto social público a distintas escalas (federal, estatal y municipal) (De la Peña, 2003). Para ello, es necesario acrecentar las investigaciones sobre los cambios en la desigual distribución espacial de la población adulta mayor y la situación de estas personas a escala de colonias y manzanas en los municipios y, sobre todo, en las áreas metropolitanas. Sus resultados nos permitirán hacer mejores diagnóstico y propuestas de planeación sobre el fenómeno demográfico y socioespacial, así como concebir nuevas políticas basadas en la prevención y en la atención adecuada de las necesidades del adulto mayor.

Lo anterior no sería posible sin la presencia de gerontólogos sociales, con una visión integral de la vejez, en los comités consultivos, en las organizaciones no gubernamentales (Caritas Diocesana) y en la gestión pública. Por lo general, en las ciudades predomina un enfoque de planeación gerontológica con tendencia normativa, lo que explica que en la mayoría de los casos la ejecución de las estrategias municipales planificadas no satisfacen las necesidades reales y heterogéneas de las personas adultas mayores. La mayor presencia y reconocimiento social del papel del gerontólogo social a nivel mundial, especialmente en México, debe contribuir a la puesta en marcha de alternativas de planeación gerontológica en las grandes urbes.

\section{Alternativas a la planeación gerontológica urbana}

En la actualidad existen diferentes propuestas en materia de planeación gerontológica de los espacios urbanos que pasamos a describir (Sánchez, 2005a):

- Promover la participación social de las personas adultas mayores a través de las asociaciones de mayores y aprovechar su experiencia en los procesos de planeación urbana con objeto de proponer alternativas para mejorar la habitabilidad de las ciudades.

- Impulsar una planeación estratégica del bienestar de las personas mayores mediante el conocimiento global de la realidad vivencial y heterogénea de los adultos mayores a distintas escalas regional, municipal y metropolitana (colonia, manzana), adecuando las acciones a la realidad de cada espacio. 
- Elaborar un sistema integral de información a partir de diagnósticos de la realidad territorial intraurbana, metropolitana y regional de los adultos mayores y el empleo de Sistemas de Información Geográficas.

- Establecer propuestas de planeación urbana y territorial (medio ambiente, estructura urbana y vivienda, infraestructura primaria, vialidad y transporte público, servicios sociales y sanitarios) para el desarrollo y mejora de la habitabilidad urbana de este sector de población heterogéneo.

- Favorecer la mayor participación y coordinación de los distintos agentes sociales implicados en el mundo del adulto mayor urbano, tanto las distintas instituciones gubernamentales (municipio, estado) como las no gubernamentales (Caritas Diocesana, Cruz Roja, asociaciones vecinales, etc.).

- Proponer alternativas integrales frente a las necesidades de las personas mayores, para favorecer el uso correcto y eficaz de los recursos materiales y humanos disponibles.

- Ayudar al desarrollo de una conciencia crítica de las personas mayores que permita transformar constructivamente la sociedad y posibilite una mayor integración social.

Es necesario que la planeación gerontológica urbana se realice potenciando el uso de metodologías cuantitativas y cualitativas de evaluación y participación social, como las encuestas, entrevistas personales y los talleres comunitarios, así como el empleo de la estadística multifactorial y los Sistemas de Información Geográficos. Las autoridades municipales y metropolitanas serán las encargadas de atender las necesidades de la creciente población heterogénea de 60 y más años en el marco de las grandes ciudades. En este sentido el sector público y los investigadores deben promover líneas de investigación que fomenten el desarrollo social y asistencial de la población adulta mayor, fortaleciendo la formación de recursos hu- manos capaces de atender las necesidades de este sector de población heterogéneo.

\section{Conclusión}

En México la escasa comprensión del proceso de envejecimiento demográfico urbano a nivel regional, municipal y metropolitano está generando decisiones políticas equivocadas. Las previsiones para las próximas décadas prevén un avance del envejecimiento de la población con marcadas diferencias territoriales, y presentando un modelo polarizado basado en la concentración urbana y en la dispersión rural de los adultos mayores. Se desprende que los mayores retos sobre el fenómeno demográfico los enfrentarán los municipios urbanos y, sobre todo, las áreas metropolitanas que hoy concentran a casi uno de cada dos mexicanos (48,4 millones de personas).

Las escalas urbanas (municipal y metropolitana) nos permiten observar la problemática situación de vulnerabilidad de los adultos mayores (pobreza, sin derecho a seguro médico y social, vivienda y soledad) que hoy residen en las principales ciudades del país. Aquí, los factores socioeconómicos (educación, profesión) durante la vida laboral condicionan la situación del adulto mayor en la vejez (seguro médico, vivienda). Precisamente, estos indicadores de vulnerabilidad en la vejez presentan notables diferencias a escala metropolitana. Así, se observa que las regiones más dinámicas y menos envejecidas (localidades y áreas metropolitanas de atracción) del país, ubicadas en la zona centro (Toluca) y norte (Monterrey), se registran menores tasas de adultos mayores en riesgo de vulnerabilidad. En tanto que las regiones menos dinámicas y envejecidas (localidades y áreas metropolitanas de expulsión), ubicadas en regiones del sur y afectadas por la emigración (Oaxaca y Tuxtla Gutiérrez), se incrementa el riesgo de padecer vulnerabilidad entre los adultos mayores.

En los próximos años la planeación gerontológica debe ser prioritaria en las políticas regionales, municipales y metropolitanas de la vejez, propiciando perspectivas adecuadas de calidad de vida a los adultos mayores (salud, dependencia, familia, socie- 
dad, economía, vivienda) en un marco espacial de referencia tan complejo y dinámico como la ciudad. Es necesario que se favorezca la planeación urbana desde una óptica integradora y gerontológica, así como el bienestar de la población y su crecimiento sostenible.

Hoy en México los municipios y, en mayor medida, las áreas metropolitanas no están preparados para asumir el reto del envejecimiento demográfico urbano y atender las necesidades y demandas de las personas adultas mayores. En cuatro décadas en este país uno de cada cuatro mexicanos tendrá 60 y más años, lo que podría ser dramático para muchos Ayuntamientos que hoy día tienen importantes rezagos en materia de servicios sociales. Todo ello puede provocar un colapso en el sistema de respuesta social, médica y asistencial de las ciudades. Es necesario ampliar la cobertura de los programas sociales municipales de la tercera edad, muy limitados en la actualidad. La inquietud no radica en que en las próximas décadas haya más ancianos en nuestras ciudades, sino generar las condiciones de bienestar para lograr que en la etapa de la vejez las personas de edad estén sanas, activas y participativas. Además, es importante que se produzca un pacto a nivel municipal y metropolitano por el adulto mayor, donde participen los profesionales, las autoridades municipales y la ciudadanía (adultos mayores y sus familias). Como última reflexión tomo el escrito de García Márquez, en el año 2000, sobre los millones de adultos mayores que viven en nuestras pequeñas, medianas y grandes ciudades en México y América Latina: "a los viejos les enseñaría que la muerte no llega con la vejez, sino con el olvido".

*Quiero expresar mi agradecimiento a la Lic. Alma Lilia Olvera Hernández y a Jimena Sánchez Olvera.

\section{Referencias bibliográficas}

BOLTVINIK, J. y DAMIÁN, A. La pobreza ignorada. Evolución y características. Papeles de Población, 2001, № 29, p. 21-53.

CASTELLS, M. La cuestión urbana. México: Siglo XXI Editores, 1978.
CASTILLO, F. y VELA, F. Envejecimiento demográfico en México. Evaluación de los datos censales por edad y sexo, 1970-2000. Papeles de Población, 2005, № 45 p. 107-141.

COMPÁN, D. y SÁNCHEZ, D. Los ancianos al desván. El proceso de degradación biológica y social de la población mayor del municipio de Granada. Cuadernos Geográficos de la Universidad de Granada, 2005, Vol. 36, № 1, p. 255-274.

CONAPO. Proyecciones de la población en México 2000-2050. México: Consejo Nacional de Población, 2002.

DE LA PEÑA, J. I. Impacto del envejecimiento de la población en el seguro de salud y la dependencia. Papeles de Población, 2003, № 35, p. 47-78.

GILBERT, A. \& GUGLeR, J. Cities, Poverty and Development, Urbanization in the Third Word. Oxford: Oxford University Press, 1992.

GUTIÉRREZ, L. y GARCÍA, E. Salud y envejecimiento de la población en México. Mexico City: Department of Economic and Social Affairs United Nations Secretariat, 31 August - 2 September, 2005.

HARVEY, D. Social Justice and the City. London: Rout ledge and Keg a Paul editors, 1973.

HELPAGE INTERNACIONAL. Estado mundial de las personas mayores 2002. Londres: Helpage Internacional, 2002.

INEGI. Indicadores sociodemográficos de México (1930-2000). México: Instituto Nacional de Estadística, Geografía e Informática, 2000.

INEGI. XII Censo General de Población y Vivienda 2000. México: Instituto Nacional de Estadística, Geografía e Informática, 2000 .

INEGI. Los adultos mayores en México. Perfil sociodemográfico al inicio del siglo XXI. México: Instituto Nacional de Estadística, Geografía e Informática, 2005. 
INEGI. I/ Conteo de población y vivienda 2005. México: Instituto Nacional de Estadística, Geografía e Informática, 2005.

INSERSO. Plan Gerontológico. Madrid: Instituto de Servicios Sociales, 1993.

INSTITUTO NACIONAL DEL ENVEJECIMIENTO: Envejecimiento en las Américas. Proyecciones para el siglo XXI. Nueva York: Departamento de Comercio de los EE.UU., 2000.

JACKSON, R. Building human capital in an aging Mexico. A report of the US - Mexico binational council. México: Instituto Tecnológico Autónomo de México, 2005.

KENNEDY, J. M. \& DEJONG, D. F. Aged in cities: residential segregation in the USA central cities. Journal of Gerontology, 1977.

KINSELLA, K. \& VELKOFF, V. A. An Aging World: 2001. Washington: U.S. Government Printing Office, 2002.

MENDOZA, D. Los factores determinantes de la disminución de la fecundidad. Los efectos de la urbanización, salud, educación y trabajo femenino. Demos, Carta demográfica sobre México, 1998, № 11.

MENÉNDEZ, J. Population aging and the labour market: issues relevant for social security in Mexico. 4th International Research Conference on Social Security. International Social Security Association, Antwerp, 2003, 5-7 May.

MONTES DE OCA, V. El envejecimiento demográfico en el debate mundial: Reflexión académica y política. Papeles de Población, 2003, № 35, p. 79-104.

MONTES DE OCA, V. Primer Seminario Sobre los Adultos Mayores en el Distrito Federal. Ciudad de México, 1998, 22 octubre.

MUÑOZ, F. Le déclin de la fécondité dans le sud de l'Europe. Population, 1987, Vol. 42, № 6, p. 911-942.
NEGRETE, M. E. Distribución geográfica de la población mayor. Demos, Carta demográfica sobre México, 2001, № 29.

NEGRETE, M. E. El envejecimiento poblacional en la ciudad de México: evolución y pautas de distribución espacial entre 1970-2000. Papeles de Población, 2003, № 37 , p. 5-27.

OATES, W. Fiscal Federalism. New York: Harcourt Brace Janovitch, 1972.

ONU. Periodical on aging. New York: Department of International Economic and Social Affairs, United Nations, 1985.

PARTIDA, V. La transición demográfica y el proceso de envejecimiento en México. Papeles de Población, 2005, № 45, p. 9-27.

ROMERO, L.; HERNÁNDEZ, M. y ACEVEDO, J. Vivienda y autoconstrucción: participación femenina en un proyecto asistido. Frontera Norte, 2005, Vol. 17, № 33, p. 105-131.

SALGADO, A. Planificación gerontológica intra y extrahospitalaria. Tesis doctoral. Madrid: Universidad Complutense de Madrid, 1976.

SÁNCHEZ, D. La situación de las personas mayores en la ciudad de Granada. Estudio Geográfico. Granada: Editorial Universidad de Granada, 2005a.

SÁNCHEZ, D. El proceso de envejecimiento demográfico urbano en Granada y su área Metropolitana. Cuadernos Geográficos de la Universidad de Granada, Vol. 2, № 37, 2005b, p. 185-200.

SCHTEINGART, M. y GRAIZBORD, B. Vivienda y vida urbana en la ciudad de México: la acción de Infonavit. México: El Colegio de México, 2000.

WARNES, A. M. \& HORSEY, A. Ageing among Latin American countries. Conference on Aging and Well-Being in Latin America. Gainesville: Center for Gerontological Studies, Universidad de Florida, 1988. 
